Energy di ssi pat i on decrease during adi abat i c charging of a capacitor by changing the duty rat i o

\begin{tabular}{|c|c|}
\hline 著者 & $\begin{array}{l}\text { Nakat a Shunj i, Naki no Hi roshi, Mit oh } \\
\text { Shi n' i chi ro, M yama Nasayuki, Mat suda Yoshi o }\end{array}$ \\
\hline 著者別表示 & 深山 正幸, 松田 吉雄 \\
\hline $\begin{array}{l}\text { j our nal or } \\
\text { publ i cat i on titl e }\end{array}$ & M dwest Symposi um on Ci rcuits and Systens \\
\hline page $r$ ange & 6026367 \\
\hline year & 2011-01- 01 \\
\hline URL & ht t p: //doi . or g/10. 24517/00008445 \\
\hline
\end{tabular}




\title{
Energy Dissipation Decrease During Adiabatic Charging of a Capacitor by Changing the Duty Ratio
}

\author{
Shunji Nakata $^{1}$, Hiroshi Makino ${ }^{2}$, Shin'ichiro Mutoh ${ }^{1}$, Masayuki Miyama ${ }^{3}$, and Yoshio Matsuda ${ }^{3}$ \\ ${ }^{1}$ NTT Microsystem Integration Labs, Nippon Telegraph and Telephone Corp., Atsugi, Japan: nakata.shunji@lab.ntt.co.jp \\ ${ }^{2}$ Faculty of Information Science and Technology, Osaka Institute of Technology, Osaka, Japan: makino@is.oit.ac.jp \\ ${ }^{3}$ Graduate School of Natural Science, Kanazawa University, Kanazawa, Japan: matsuda@ec.t.kanazawa-u.ac.jp
}

\begin{abstract}
Adiabatic charging of a capacitor with a step down converter by changing the duty ratio is considered. First, for a profound understanding of the circuit, the general analytical solution of step down converter is considered. It is confirmed that the system can be resolved analytically and that the equilibrium state of current and voltage are consistent with SPICE simulation. Next, adiabatic charging by changing the duty ratio is investigated. From SPICE simulation, it is confirmed that energy dissipation is reduced to one-fourth when four-step charging is used. By increasing the step number, energy dissipation decreases to zero and dissipationless operation is achieved.
\end{abstract}

\section{INTRODUCTION}

Recently, renewable energy is considered to be very important for achieving a sustainable society. In this area, energy storage is very important because the renewable energy from sources, such as wind power and solar power, is not continuously output. There are several ways to store the energy, such as lead-acid batteries, NiMH batteries, Li ion batteries, and supercapacitors. Among them, we believe that supercapacitors will become more important in future. Supercapacitors can be charged and discharged more than 100,000 times. Moreover, they can operate in the wide temperature region of -25 to $70{ }^{\circ} \mathrm{C}$, which is wider than that of $\mathrm{Li}$ ion batteries. Further, supercapacitors exhibit significant higher volumetric power density. However, circuits for charging and discharging supercapacitors have not been well studied yet.

In this article, we demonstrate that adiabatic stepwise charging [1-5] is effective for reducing the energy dissipation when using a step down converter. We have already introduced an effective technique for both charging and discharging a capacitor in the same circuit [6]. As shown in Fig. 1, when charging, the circuit operates as a step down converter and current flows from $\mathrm{X}$ to $\mathrm{Y}$. When discharging, it operates as a step up converter and current flows from $Y$ to $X$. Charging and discharging are performed by changing the duty ratio stepwise. This means that the capacitor is charged stepwise during charging and energy is recovered (or discharged) stepwise to power supply $E$ gradually.

However, in the circuit in Fig. 1, the reduction of energy dissipation has not yet been confirmed. In this article, we investigate this point in detail using SPICE simulation.

First, for a profound understanding of the circuit in Fig. 1, we analyze the general $L C R$ circuit. The voltage and current values after the circuit reaches the equilibrium state are discussed analytically in Sec. II and discussed using SPICE simulation in Sec. III. Next, using the $L C R$ circuit analysis, we perform the SPICE simulation of the adiabatic charging.

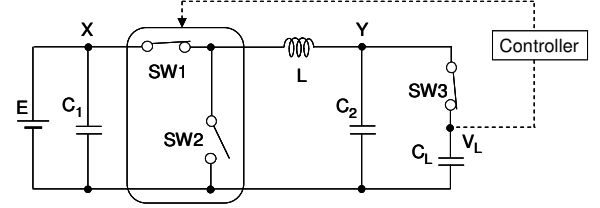

Fig. 1. A circuit that can charge and discharge a load capacitor adiabatically.

The energy dissipation is calculated when the capacitor is charged stepwise. It is clarified that, for four-step charging, energy dissipation decreases to one-fourth compared to the conventional constant voltage charging. This is described in Sec. IV.

\section{LCR CIRCUIT ANALYSIS}

For a more profound understanding of the circuit behavior in Fig. 1, we consider the general $L C R$ circuit shown in Fig. 2(a). The $E_{e x}(t)$ is the external voltage. $I$ is the current through the inductor and $V$ is the voltage of capacitor. Here, we consider a periodic square wave as $E_{e x}(t)$. Using the duty ratio $d$, a period $T$, and a constant voltage $E$, we express $E_{e x}(t)$ in a period as

$$
E_{e x}(t)=\left\{\begin{array}{ll}
E, & (0 \leq t<d T) \\
0, & (d T \leq t<T)
\end{array} .\right.
$$

This $E_{e x}(t)$ is considered to be the same as the left part of the step down converter in Fig. 2(b). Therefore, analyzing a square wave means analyzing the step down circuit in Fig. 2(b). When $0 \leq t<d T$ (mode 1 ) and $d T \leq t<T$ (mode 2), the circuit equations are written as

$$
L \frac{d I}{d t}+R I+V=E \text { and } L \frac{d I}{d t}+R I+V=0,
$$

respectively. By connecting the solutions of (2), we can resolve these differential equations. The derivation will be discussed in detail elsewhere. Then, we have the expression of the current at the beginning of mode $1, I_{n}$, as

$$
I_{n}=c_{1} e^{n k_{1} T}+c_{2} e^{n k_{2} T}+c_{3}(n),
$$

where $n$ is the number of square waves from $t=0, c_{1}$ and $c_{2}$ are constant, and $c_{3}(n)$ is a function of $n . \quad k_{1}$ and $k_{2}$ are characteristic solutions of (2) and are written as

$$
k_{1}=\frac{-R+\sqrt{R^{2}-4 L / C}}{2 L} \text { or } k_{2}=\frac{-R-\sqrt{R^{2}-4 L / C}}{2 L} .
$$

The real part of $k_{1}$ and $k_{2}$ is always negative so that $e^{n k_{1} T}$ and $e^{n k_{2} T}$ become zero when $n$ is large. Therefore, the converged value of $I_{n}, I_{f}$, is written as

$$
\begin{aligned}
& I_{f}=\lim _{n \rightarrow \infty} I_{n}=\lim _{n \rightarrow \infty} c_{3}(n) \\
& =\frac{C E k_{1} k_{2}}{k_{2}-k_{1}}\left\{\frac{\left(e^{k_{1}(1-d) T}-e^{k_{1} T}\right)}{1-e^{k_{1} T}}-\frac{\left(e^{k_{2}(1-d) T}-e^{k_{2} T}\right)}{1-e^{k_{2} T}}\right\} .
\end{aligned}
$$




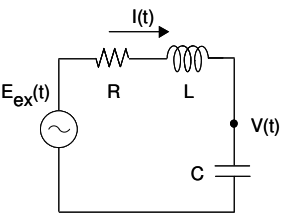

(a)

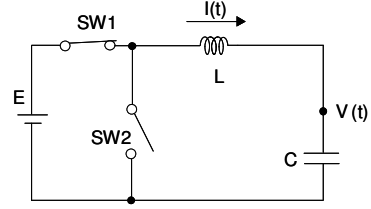

(b)
Fig. 2. (a) The general LCR circuit with external voltage $E_{e x}(t)$. (b) Step down converter. A switch is composed of nMOS and pMOS transistors.

In power electronics circuit, the switching frequency is sufficiently faster than the resonant frequency. Therefore, we have the relations $k_{1} T<<1$ and $k_{2} T<<1$. Then, using (5), we have

$$
I_{f}=-\frac{d(1-d) T E}{2 L} .
$$

Regarding the converged current at the beginning of mode 2 , $I_{f}^{\prime}$, we can derive the relation $I_{f}^{\prime}=-I_{f}$.

Regarding the voltage, we define $V_{n}$ and $V_{n}^{\prime}$ as the voltage at the beginning of modes 1 and 2, respectively. Then, we define $V_{f}$ and $V_{f}^{\prime}$ as $V_{f}=\lim _{n \rightarrow \infty} V_{n}$ and $V_{f}^{\prime}=\lim _{n \rightarrow \infty} V_{n}^{\prime}$. Then, using $k_{1} T<<1$ and $k_{2} T<<1$, we have

$$
V_{f}=V_{f}^{\prime}=d E \text {. }
$$

Equation (7) is consistent with formula in power electronics. Regarding the current, the average current in the equilibrium state is zero so that the relation $I_{f}^{\prime}=-I_{f}$ is reasonable.

\section{SimULATION OF EQUIRIBRIUM STATE}

In this section, to verify (6), we investigate the $I_{f}$ and $I_{f}^{\prime}$ of the circuit in Fig. 2(b) with SPICE. We used the 180-nm design rule. The transistor gate length $L_{g}$ is $0.18 \mu \mathrm{m}$ and gate widths of pMOS and nMOS $W_{p}$ and $W_{n}$ are 200 and $100 \mu \mathrm{m}$, respectively. $E$ is $2 \mathrm{~V}$. The gate voltage of SW1 and SW2 is changed from 0 to $3.3 \mathrm{~V}$. Threshold voltages $V_{T}$ 's are 0.43 and $-0.33 \mathrm{~V}$ in nMOS and pMOS, respectively. The body biases of nMOS and pMOS are GND and VDD, respectively.

The simulation result is shown in Fig. 3 . $L$ is 1,10 , or 20 $\mu \mathrm{H}$. $T$, which corresponds to the switching period, is set to $0.1 \mu \mathrm{s}$. The duty ratio is changed as $0.2,0.4,0.6$, and 0.8 . It is clarified that the capacitance voltage $V(t)$ increases stepwise as $0.4,0.8,1.2$, and $1.6 \mathrm{~V}$, which are consistent with $d E$. Regarding the current, the current flows largely at the beginning of each step. After large flows of current, the circuit reaches the equilibrium state. This is common with $L=1,10$, and $20 \mu \mathrm{H}$. The equilibrium current oscillates between two values. It is confirmed from Fig. 3 that the oscillation amplitude decreases when $L$ increases. Fig. 4 is a magnification of Fig. 3 at $t=154 \mu \mathrm{s}$. When $L=1 \mu \mathrm{H}$, the current oscillates linearly between -23.9 and $23 \mathrm{~mA}$. When $L=10$ and $20 \mu \mathrm{H}$, it oscillates between -2.4 and $2.4 \mathrm{~mA}$ and between -1.2 and $1.2 \mathrm{~mA}$, respectively. With $d(1-d) T E / 2 L$, the theoretical values are $24,2.4$, and $1.2 \mathrm{~mA}$, which are perfectly consistent with the SPICE simulation results. Therefore, the analytical formula is correct.

\section{Simulation OF Adiabatic Charging}

Here, we discuss the effectiveness of stepwise adiabatic charging. We set $L$ to $800 \mu \mathrm{H}$ in order to make the
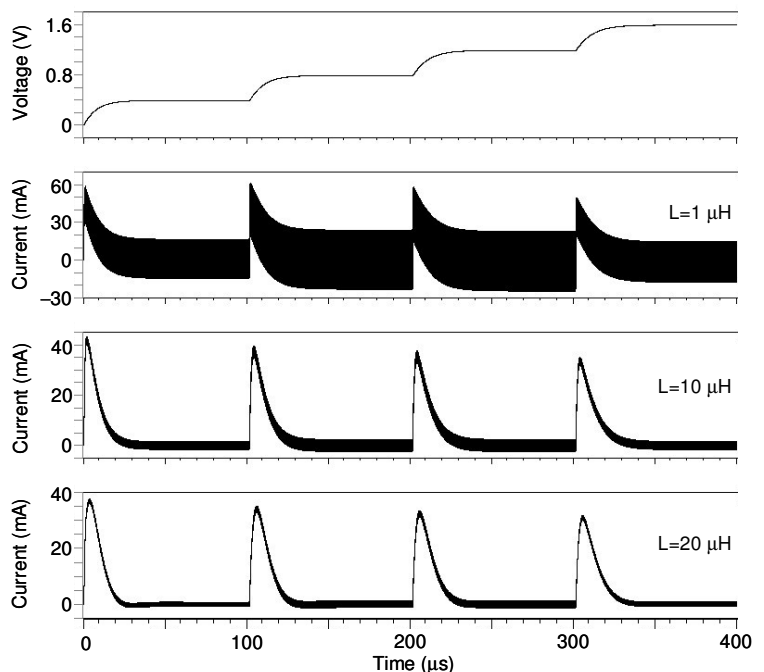

Fig. 3. $\quad V$ and $I$ of step down converter when the duty ratio is changed stepwise.
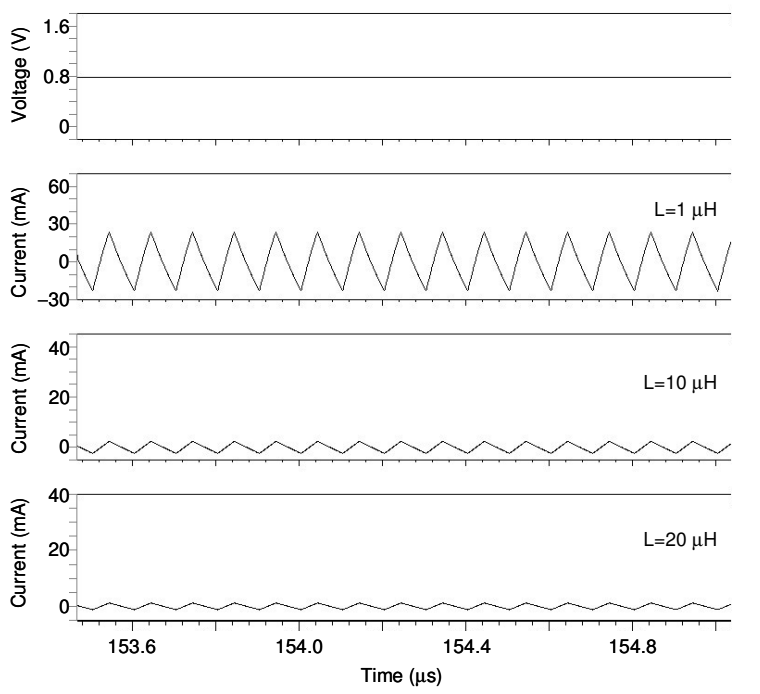

Fig. 4. Magnification of current in Fig. 3 at $t=154 \mu \mathrm{s}$.

equilibrium current oscillation almost zero. By making $I_{f}$ and $I_{f}^{\prime}$ zero, we can decrease the Joule heat at the equilibrium state. After setting $L$ to $800 \mu \mathrm{H}$, we set $C$ to $1,20,40$, or 50 $\mu \mathrm{F}$ to investigate the adiabatic charging in detail. We discuss the change of the voltage and current in each case. In the following simulations, we set $T$ to $1 \mu \mathrm{s}$.

Fig. 5 shows the simulation results for $C=1 \mu \mathrm{F}$. The time period for each step charging is $1 \mathrm{~ms}$. The average voltage almost changes stepwise. However, the voltage oscillates until it converges to the step value. Also, the current oscillates according to the $I=C d V / d t$. Due to the voltage oscillation, the voltage increases more than the expected step voltage. In particular, this voltage increase at the final step is not good for a device. We should decrease the voltage overshoot due to the oscillation. The oscillation occurs because $k_{1}$ and $k_{2}$ in (3) are complex. If $k_{1}$ and $k_{2}$ are real numbers, oscillation does not occur. To make $k_{1}$ and $k_{2}$ real numbers, the value of $R^{2}-4 L / C$ should be positive, so the $C$ value should be increased.

Fig. 6 shows the simulation results for $C=50 \mu \mathrm{F}$. The 

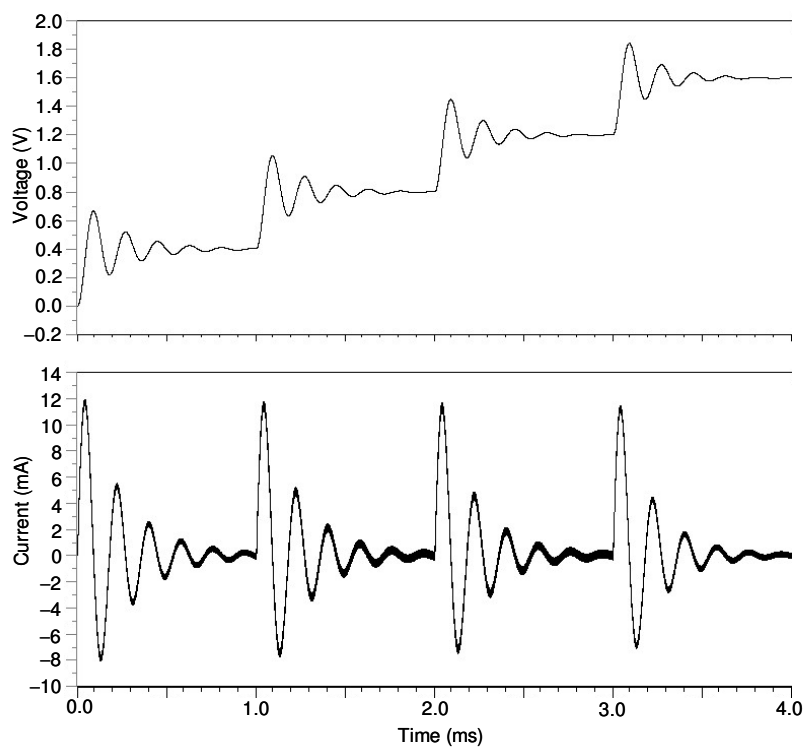

Fig. 5. $V$ and $I$ of step down converter when $L=800 \mu \mathrm{H}$ and $C=1 \mu \mathrm{F}$.
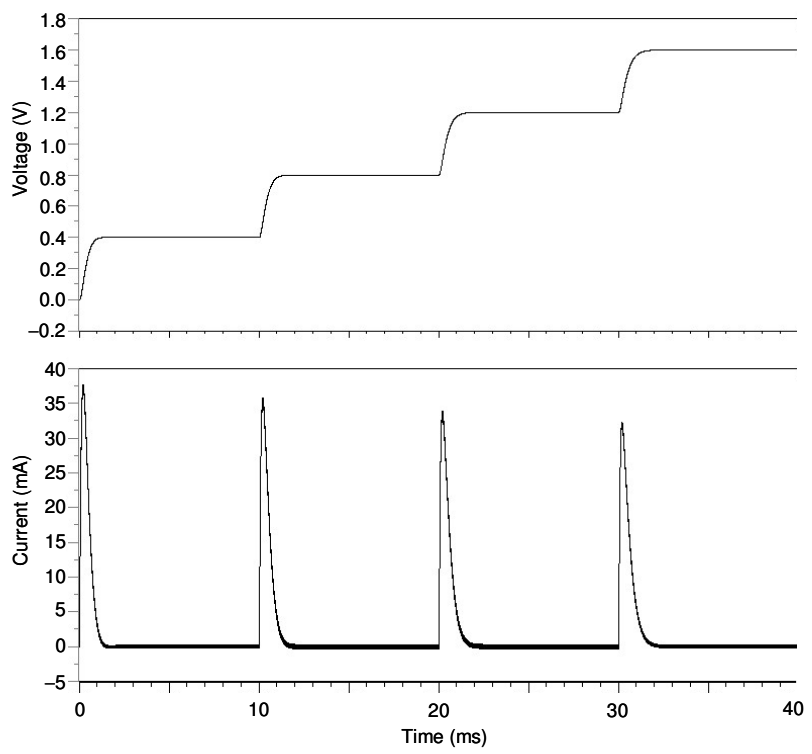

Fig. 6. $V$ and $I$ of step down converter when $L=800 \mu \mathrm{H}$ and $C=50 \mu \mathrm{F}$.

time period for each step charging is $10 \mathrm{~ms}$. In this case, the oscillation and the overshoot completely disappear. Regarding the current, it increases largely at the beginning and then decreases monotonically. This behavior is close to conventional $R C$ circuit charging.

Due to the disappearance of the oscillation, when $C=50$ $\mu \mathrm{F}, R^{2}-4 L / C=0$ should be satisfied. Then, $R$ is estimated to be $8 \Omega$. This value is consistent with the resistance of the switching transistor.

In the equilibrium state at $t=19 \mathrm{~ms}$, the current changes linearly between -0.3 and $0.3 \mathrm{~mA}$. The $-0.3 \mathrm{~mA}$ is also quite consistent with (6).

Now, we discuss the energy dissipation. Table I shows the power consumption $P$ and the injected energy for charging the capacitor $E_{i n j}$ by the simulation. $L$ is $800 \mu \mathrm{H}$ and $C$ is 20,40 , or $50 \mu \mathrm{F}$. The duty ratio is changed as $0.2,0.4$, 0.6 , or 0.8 . First, we discuss the situation when $d=0.2$ and
Table I. Power consumption and injected energy for charging the capacitor of step down converter when the duty ratio is changed stepwise.

\begin{tabular}{|c|l|c|c|c|}
\hline \multirow{3}{*}{$d$} & \multicolumn{1}{|c|}{$P$ and $E_{\text {inj }}$} & \multicolumn{3}{|c|}{ Simulation value } \\
\cline { 3 - 5 } & & $L$ & \multicolumn{3}{|c|}{$800 \mu \mathrm{H}$} \\
\hline \multirow{4}{*}{0.2} & $C$ & $20 \mu \mathrm{F}$ & $40 \mu \mathrm{F}$ & $50 \mu \mathrm{F}$ \\
\cline { 2 - 5 } & Ave. $P[\mathrm{~mW}] 0 \sim 10 \mathrm{~ms}$ & 0.342 & 0.660 & 0.819 \\
\cline { 2 - 5 } & Ave. $P[\mathrm{~mW}] 9 \sim 10 \mathrm{~ms}$ & 0.023 & 0.023 & 0.023 \\
\cline { 2 - 5 } & $E_{i n j}$ for charging $C[\mathrm{~nJ}]$ & 3190 & 6370 & 7960 \\
\hline \multirow{4}{*}{0.4} & Ave. $P[\mathrm{~mW}] 10 \sim 20 \mathrm{~ms}$ & 0.663 & 1.302 & 1.622 \\
\cline { 2 - 5 } 0.6 & Ave. $P[\mathrm{~mW}] 19 \sim 20 \mathrm{~ms}$ & 0.024 & 0.024 & 0.024 \\
\cline { 2 - 5 } & $E_{i n j}$ for charging $C[\mathrm{~nJ}]$ & 6390 & 12780 & 15980 \\
\hline \multirow{3}{*}{0.8} & Ave. $P[\mathrm{~mW}] 20 \sim 30 \mathrm{~ms}$ & 0.983 & 1.942 & 2.422 \\
\cline { 2 - 5 } & Ave. $P[\mathrm{~mW}] 29 \sim 30 \mathrm{~ms}$ & 0.023 & 0.023 & 0.023 \\
\cline { 2 - 5 } & $E_{i n j}$ for charging $C[\mathrm{~nJ}]$ & 9600 & 19190 & 23990 \\
\hline \multirow{3}{*}{0.8} & Ave. $P[\mathrm{~mW}] 30 \sim 40 \mathrm{~ms}$ & 1.303 & 2.582 & 3.222 \\
\cline { 2 - 5 } & Ave. $P[\mathrm{~mW}] 39 \sim 40 \mathrm{~ms}$ & 0.023 & 0.023 & 0.023 \\
\cline { 2 - 5 } & $E_{i n j}$ for charging $C[\mathrm{~nJ}]$ & 12800 & 25590 & 31900 \\
\hline
\end{tabular}

$C=20 \mu \mathrm{F}$. The average power consumption for $t=0$ to $10 \mathrm{~ms}$ is $0.342 \mathrm{~mW}$. This value includes the power consumption of charging the capacitor and the steady power consumption of the circuit. The average power consumption for $t=9$ to $10 \mathrm{~ms}$ is $0.023 \mathrm{~mW}$. We can confirm that the average power consumption for $t=8$ to $9 \mathrm{~ms}$ is also $0.023 \mathrm{~mW}$. Therefore, it is concluded that $0.023 \mathrm{~mW}$ corresponds to the steady power consumption of the circuit. Then, we can estimate the injected energy for charging the capacitor $E_{i n j}$ during the first step. $E_{i n j}$ is consistent with the work done by the power supply. The total energy consumption during the first step is $3420 \mathrm{~nJ}$. The steady energy consumption of the circuit during the first step is $230 \mathrm{~nJ}$. Therefore, $E_{i n j}$ for charging the capacitor is $3190 \mathrm{~nJ}$. The theoretical $E_{i n j}$ for charging the capacitor with constant power supply voltage $d E$ is $d E \Delta Q$, where $\Delta Q$ is the stored charge difference in the capacitor. Then, when $d=0.2, \Delta Q$ is written as $0.2 E C$. Therefore, $d E \Delta Q$ is calculated as $3200 \mathrm{~nJ}$, which is consistent with the simulated value.

The simulated values for $d=0.4,0.6$, and 0.8 are shown in Table I. Then, $E_{i n j}$ values for charging the capacitor are similarly calculated as 6390,9600 , and $12800 \mathrm{~nJ}$ for $d=0.4$, 0.6 , and 0.8 , respectively. The theoretical $d E \Delta Q$ values are 6400,9600 , and $12800 \mathrm{~nJ}$, which are consistent with the simulated ones. Now, we consider the energy efficiency of stepwise adiabatic charging. The total $E_{i n j}$ for charging the capacitor is the sum of $3190,6390,9600$, and $12800 \mathrm{~nJ}$, which is $31980 \mathrm{~nJ}$. The static electric energy of the capacitor is $C V^{2} / 2$, which is calculated as $1 / 2 \cdot 20 \mu \mathrm{F} \cdot(1.6 \mathrm{~V})^{2}=25600 \mathrm{~nJ}$. The energy dissipation is the difference between the total $E_{i n j}$ for charging the capacitor and the capacitor's static electric energy. Therefore, the energy dissipation is $31980-25600=6380 \mathrm{~nJ}$. In the conventional constant voltage charging, the energy dissipation is equal to $C V^{2} / 2,25600 \mathrm{~nJ}$. Then, the energy dissipation decreases to $6380 / 25600=24.9 \%$, which is consistent with the theoretical value of one-fourth for four-step charging. We can also confirm that energy dissipation decreases to one-fourth when $C=40$ and $50 \mu \mathrm{F}$.

Next, we consider the circuit with large load capacitance $C_{1}$ as shown in Fig. 7. Large capacitance of $100 \mu \mathrm{F}$ is connected to the output voltage of the step down converter via resistor $R_{1}$. This circuit topology is conventionally used for the step down converter. The simulation of voltage and current is shown in Fig. 8. Here, $C$ is set to $1 \mu \mathrm{F}$. While the 


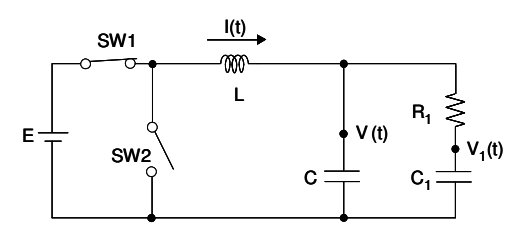

Fig. 7. Step down circuit with large load capacitance $C_{1}$.
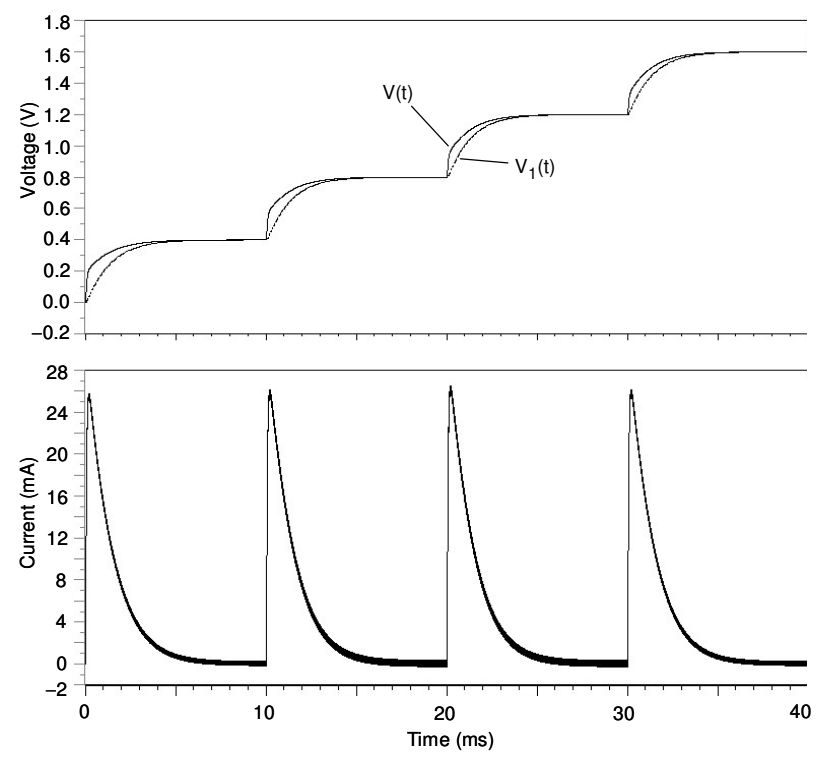

Fig. 8. $V, V_{1}$, and $I$ of the circuit in Fig. 7. $C$ and $C_{1}$ are 1 and $100 \mu \mathrm{F}$, respectively.

voltage in Fig. 5 oscillates largely, the voltage in Fig. 8 does not oscillate due to the large load capacitance, although these circuits have the same $C$ of $1 \mu \mathrm{F}$.

Now, we consider the energy efficiency. Table II shows the simulation results in Fig. 8. When $C=1 \mu \mathrm{F}, E_{i n j}$ values for charging the load capacitor are 16010, 32140, 48300, and $64360 \mathrm{~nJ}$ for $d=0.2,0.4,0.6$, and 0.8 , respectively. The theoretical values are 16160, 32320, 48480, and $64640 \mathrm{~nJ}$, which are consistent with the simulated ones. Now, we consider the energy efficiency of stepwise adiabatic charging. The total $E_{i n j}$ for charging the load capacitor is $160810 \mathrm{~nJ}$. The $C V^{2} / 2$ is calculated as $129280 \mathrm{~nJ}$. Therefore, the energy dissipation of stepwise charging is $31530 \mathrm{~nJ}$. As a result, the energy dissipation decreases to $31530 / 129280=24.4 \%$, which is consistent with the theoretical value of one-fourth. Similarly, we can confirm that, when $C=10 \mu \mathrm{F}$, the energy dissipation decreases to $24.2 \%$, which is also consistent with the theoretical value.

In stepwise charging, energy efficiency is related to the number of steps. When we increase $n$, energy dissipation would become zero.

Here, we consider $E_{i n j}$ for charging the gate capacitance of SW1 and SW2 in Fig. 2(b) or Fig. 7. The simulation results are $0.7,0.6,0.6,0.7 \mu \mathrm{W}$ for $d=0.2,0.4,0.6$, and 0.8 , respectively. The gate capacitance per area is $1 \mathrm{fF} / \mu \mathrm{m}^{2}$ so that the gate capacitance of SW1 is estimated to be $0.18 \cdot(100+200) \mathrm{fF}=54 \mathrm{fF}$. Then, power consumption $f C V^{2}$ is calculated as $10^{6} \cdot 54 \times 10^{-15} \cdot(3.3)^{2}=0.59 \mu \mathrm{W}$. This value is consistent with the simulated value. From $t=0$ to $10 \mathrm{~ms}$, the energy consumption of the SW1 gate is $5.9 \mathrm{~nJ}$. This value is
Table II. Power consumption and injected energy for charging the capacitor in the circuit with large load capacitance of $100 \mu \mathrm{F}$.

\begin{tabular}{|c|c|c|c|c|}
\hline \multirow{3}{*}{$d$} & \multirow{3}{*}{$P$ and $E_{i n j}$} & & \multirow{2}{*}{\multicolumn{2}{|c|}{$\begin{array}{c}\text { Simulation value } \\
800 \mu \mathrm{H}\end{array}$}} \\
\hline & & $L$ & & \\
\hline & & $C$ & $1 \mu \mathrm{F}$ & $10 \mu \mathrm{F}$ \\
\hline \multirow{3}{*}{0.2} & \multicolumn{2}{|l|}{ Ave. $P(\mathrm{~mW}) 0 \sim 10 \mathrm{~ms}$} & 1.631 & 1.774 \\
\hline & \multicolumn{2}{|l|}{ Ave. $P(\mathrm{~mW}) 9 \sim 10 \mathrm{~ms}$} & 0.030 & 0.032 \\
\hline & \multicolumn{2}{|l|}{$E_{i n j}$ for charging $C[\mathrm{~nJ}]$} & 16010 & 17420 \\
\hline \multirow{3}{*}{0.4} & \multicolumn{2}{|l|}{ Ave. $P(\mathrm{~mW}) 10 \sim 20 \mathrm{~ms}$} & 3.251 & 3.539 \\
\hline & \multicolumn{2}{|l|}{ Ave. $P(\mathrm{~mW}) 19 \sim 20 \mathrm{~ms}$} & 0.033 & 0.037 \\
\hline & \multicolumn{2}{|l|}{$E_{i n j}$ for charging $C[\mathrm{~nJ}]$} & 32140 & 35020 \\
\hline \multirow{3}{*}{0.6} & \multicolumn{2}{|l|}{ Ave. $P(\mathrm{~mW}) 20 \sim 30 \mathrm{~ms}$} & 4.868 & 5.299 \\
\hline & \multicolumn{2}{|l|}{ Ave. $P(\mathrm{~mW}) 29 \sim 30 \mathrm{~ms}$} & 0.038 & 0.045 \\
\hline & \multicolumn{2}{|l|}{$E_{i n j}$ for charging $C[\mathrm{~nJ}]$} & 48300 & 52540 \\
\hline \multirow{3}{*}{0.8} & \multicolumn{2}{|l|}{ Ave. $P(\mathrm{~mW}) 30 \sim 40 \mathrm{~ms}$} & 6.483 & 7.059 \\
\hline & \multicolumn{2}{|l|}{ Ave. $P(\mathrm{~mW}) 39 \sim 40 \mathrm{~ms}$} & 0.047 & 0.058 \\
\hline & \multicolumn{2}{|l|}{$E_{i n j}$ for charging $C[\mathrm{~nJ}]$} & 64360 & 70010 \\
\hline
\end{tabular}

negligible compared with the $3190 \mathrm{~nJ}$ at $C=20 \mu \mathrm{F}$ in Table I or $16010 \mathrm{~nJ}$ at $C=1 \mu \mathrm{F}$ in Table II so that we can neglect $E_{i n j}$ of the gate capacitance.

In Fig. 8, the period of the current peak is $10 \mathrm{~ms}$ so that the region of zero current is long. Of course, we can shorten the time period to $2 \mathrm{~ms}$, in which the current does not decrease to zero and would go to the next peak. This charging method is good for high-speed operation. By decreasing the time period for step charging and increasing the step number, the stepwise charging becomes something close to constant current charging.

\section{CONCLUSION}

We investigated a step down converter with an inductor and capacitor analytically. From the analytical solution, it is derived that the voltage and current in the equilibrium state is $d E$ and $d(1-d) T E / 2 L$, where $d, E, T$, and $L$ are the duty ratio, power supply voltage, switching period, and inductance. Next, we discussed adiabatic stepwise charging, which is achieved by changing the duty ratio stepwise in the step down converter. Simulations clarified that the energy dissipation decreases to one-fourth when four-step charging is performed The situation when there is large load capacitance was also simulated, and it is clarified that the energy dissipation decreases to one-fourth.

\section{REFERENCES}

[1] R. C. Merkle, "Reversible electronic logic using switches," Nanotechnology, vol. 4, no. 1, pp. 21-40, Jan. 1993.

[2] L. J. Svensson and J. G. Koller, "Driving a capacitive load without dissipating $f C V^{2}$," in Proc. IEEE Symp. Low Power Electron., 1994, pp. 100-101.

[3] W. C. Athas, "Energy-Recovery CMOS," in Low Power Design Methodologies, J. M. Rabey and M. Pedram, Ed. Massachusetts: Kluwer Academic Publishers, 1996, pp. 65-100.

[4] S. Nakata, "Adiabatic charging reversible logic using a switched capacitor regenerator," IEICE Trans. Electron., vol. E87-C, no. 11, pp. 1837-1846, Nov. 2004.

[5] S. Nakata, H. Suzuki, R. Honda, T. Kusumoto, S. Mutoh, H. Makino, M. Miyama, and Y. Matsuda, "Adiabatic SRAM with a shared access port using a controlled ground line and step-voltage circuit," in Proc. IEEE ISCAS, 2010, pp. 2474-2477.

[6] S. Nakata, M. Miyama, and Y. Matsuda, "An adiabatic charging and discharging method with minimum energy dissipation for a variablegap capacitor system," IET Circuits, Devices and Systems, vol. 4, no. 4, pp.301-311, July 2010. 